\title{
Fifteen DO, PG 1159 and related white dwarf stars in the SDSS, including two DO stars with ultra-high excitation ion lines
}

\author{
J. Krzesiński ${ }^{1,2}$, A. Nitta ${ }^{1}$, S. J. Kleinman ${ }^{1}$, H. C. Harris ${ }^{3}$, J. Liebert ${ }^{4}$, G. Schmidt ${ }^{4}$, D. Q. Lamb ${ }^{5}$, and J. Brinkmann \\ 1 New Mexico State University, Apache Point Observatory, PO Box 59, Sunspot, NM 88349, USA \\ 2 Mt. Suhora Observatory, Cracow Pedagogical University ul. Podchorążych 2, 30-084 Cracow, Poland \\ 3 US Naval Observatory, PO Box 1149, Flagstaff, AZ 86002-1149, USA \\ ${ }^{4}$ Steward Observatory, University of Arizona, 933 N. Cherry Ave. Tucson, AZ 85721-0065, USA \\ 5 Department of Astronomy and Astrophysics, University of Chicago, 5640 South Ellis Avenue, Chicago, IL 60637, USA
}

Received 18 November 2003 / Accepted 8 January 2004

\begin{abstract}
We report on observations of 15 spectroscopically-identified DO stars from the Sloan Digital Sky Survey (SDSS) database, 13 of which are new discoveries. There are four PG 1159 type stars, two DO stars showing ultra-high excitation ion features $\left(\mathrm{C}_{\mathrm{VI}}, \mathrm{N}_{\mathrm{VII}}, \mathrm{O}_{\mathrm{VII}}, \mathrm{O}_{\mathrm{VIII}}, \mathrm{Ne}_{\mathrm{IX}}, \mathrm{Ne}_{\mathrm{X}}\right)$ likely formed in stellar winds, 6 normal DO stars, one DBO and DBAO star, and one DAO star, which may also be magnetic. Since roughly $60 \mathrm{DO}$ stars were known up to now, this new finding substantially increases the number of known DO white dwarf stars and we expect to at least double the current number of known DO stars by the end of the SDSS. We present each spectrum and provide catalog information (magnitudes, proper motion, coordinates) for each star.
\end{abstract}

Key words. stars: white dwarfs - techniques: spectroscopic - line: identification

\section{Introduction}

The DO stars are the hottest and therefore, fastest evolving white dwarf stars. Their evolutionary time scale is of order $10^{6}$ years (O'Brien \& Kawaler 2000). They have a mean mass of $0.6 M_{\odot}$, similar to the mean mass of all DA white dwarfs (Dreizler \& Werner 1996) and absolute luminosities reaching $100 L_{\odot}$ or more (Barstow \& Holberg 1990, see Fig. 3 in their paper). Because of their rapid evolution, the DO stars are relatively rare compared to most other white dwarf stars. There are only 59 spectroscopically identified known DO stars (including 18 PG 1159 type stars) as of the last published McCook \& Sion (1999). The evolution of the DO stars is primarily a cooling process where the dominant fraction of the energy is carried away by neutrinos (O'Brien \& Kawaler 2000). Their surface gravities are lower then average white dwarf stars with $\log g \approx 6-7.5$ and they have effective temperatures between $45000-150000 \mathrm{~K}$ or higher, placing them in the H-R diagram between the cool end of planetary nebulae nuclei branch and the top of the white dwarf cooling track.

Using SDSS colors alone, all normal hot white dwarf and subdwarf stars appear similar. Thus, in order to distinguish the DOs from other types of stars that have similar colors, we have to examine their spectra. The present Data Relese One (DR1) (Abazajian et al. 2003) of the Sloan Digital Sky Survey (York

Send offprint requests to: J. Krzesiński,

e-mail: jurek@apo.nmsu.edu et al. 2000) data includes $\approx 2500$ white dwarf stars (Kleinman et al. 2004), with 13 of them classified as DO stars ${ }^{1}$. Here, we include 2 additional DO stars not included in the DR1 release and provide details of the entire DO sample to date. Our classification criteria are based on the philosophy used in Sion (1983) and Wesemael (1993) where a white dwarf can be classified as a DO star only if its spectrum shows a dominant $4686 \AA \mathrm{He}_{\text {II }}$ line. Following the Wesemael et al. (1993) suggestion that there could be a cooler DO related class of helium-rich star, with $T_{\text {eff }}$ below $45000 \mathrm{~K}$, which might be called DBO, in which both $\mathrm{He}_{\mathrm{I}}$ and $\mathrm{He}_{\mathrm{II}}$ lines appeared, but $\mathrm{He}_{\mathrm{I}}$ lines are of greater strength than the strongest $\mathrm{He}_{\mathrm{II}}$ line, we called such stars similar way. Then, if a star showed hydrogen Balmer series lines in addition to $\mathrm{He}_{\mathrm{II}}$ lines, (although usually only the $4686 \AA$ line), the star was classified as a DAO or DBAO if $\mathrm{He}_{\mathrm{I}}$ lines were also present and were stronger than Balmer series lines.

\section{Target selection and observations}

Given the DR1 sample of 13 DO stars from $\approx 1500 \mathrm{deg}^{2}$, we wonder how complete is this sample? To answer this question, one needs to first understand the way the targeting for SDSS spectroscopic observations works. First, photometric observations in 5 SDSS filters $(u, g, r, i, z)$ are taken by scanning the sky

\footnotetext{
1 An additional object was classified as an uncertain DBO in Kleinman et al.'s (2004) catalogue.
} 


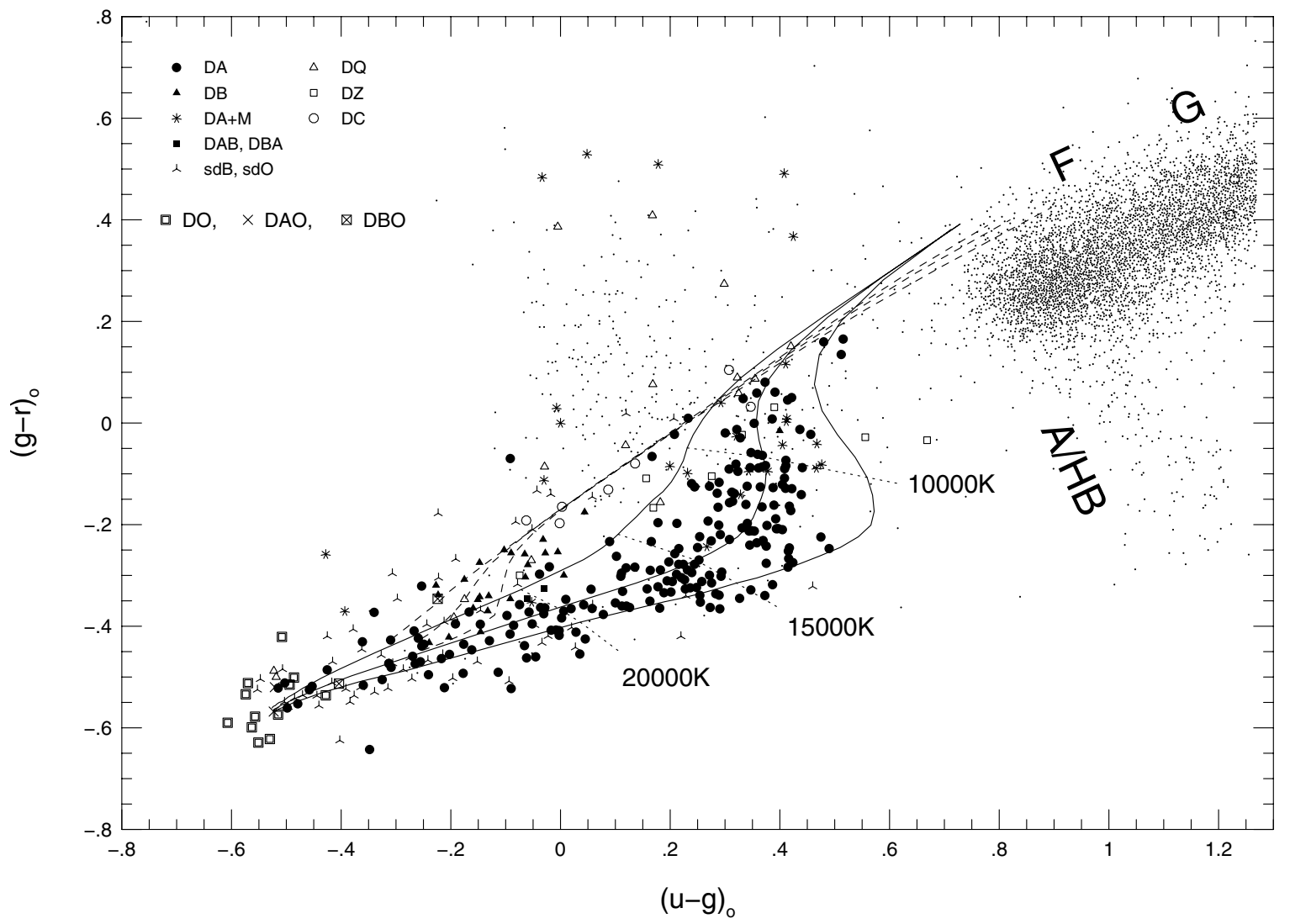

Fig. 1. Derredened color-color diagram in SDSS filters for the region occupied by the white dwarf, subdwarf and pre-white-dwarf stars. The 16 stars reported here cluster at the bottom left corner of the diagram (DOs being represented by thick lined squares; DAOs, by crosses; and DBOs, by crossed squares). Bergeron (1995) models of cooling sequences for the DA and DB white dwarf stars (convolved with SDSS filter curves) are shown as solid and dashed lines respectively, with the approximate temperature indicated at several points. The letters $F$ and $G$ show the stellar locus position and the rough $\mathrm{F}$ and $\mathrm{G}$ star positions within it. A/HB denotes the location of A type stars and the horizontal branch. Small dots are other field stars.

with a mosaic CCD camera attached to the $2.5 \mathrm{~m}$ telescope at Apache Point Observatory (APO) in New Mexico (Gunn et al. 1998; Fukugita et al. 1996; Lupton et al. 1999). Then, based on color criteria (and perhaps proper motion and other catalog cross-referencing), the selection of targets for spectroscopic fibers is made (see, Harris et al. 2003 for a short description), and the follow-up spectroscopic observations are taken with the two SDSS $2.5 \mathrm{~m}$ APO multi-fiber spectrographs ${ }^{2}$. The resulting data are low resolution $(R=1800)$ flux-calibrated spectra covering the range between 3800 to $9200 \AA$. In this paper we concentrate on a smaller overall range of wavelength, from 3800 to $6650 \AA$, where the most important DO features are present. All but one object described here have been targeted in the survey for spectroscopic observations as hot standard stars (the HOT_STD selection criteria). An object is targeted as a hot standard if its observed $g$ filter magnitude is greater than $14 \mathrm{mag}$ and its dereddened $g$ magnitude (see Schlegel et al. 1998, for the dereddening formula used by the SDSS) is less than 19 mag. In addition, its reddening corrected $u-g$ and $g-r$ colors must fall between -1.5 and 0.0 mag.

\footnotetext{
${ }^{2}$ See http://www.astro.princeton.edu/PB00K/spectro/ spectro.htm
}

To illustrate how well white dwarf target selection from photometric observations can be made, we present a SDSS color-color diagram (Fig. 1), similar to the one presented by Harris et al. (2003) with all our DO stars added. The SDSS colors are dereddened observed PSF magnitudes where the dereddening was done using the formulae given by Schlegel (1998). Two sets of lines, solid and dashed, for the DA and DB stars respectively, are the white dwarf cooling sequences calculated by Bergeron et al. (1995) and convolved with SDSS filter curves (Bergeron, private communication). Figure 1 clearly shows that all our DO stars lie well inside the hot standard region described earlier. Since the hot standard selection criteria gets allocated fibers with a very high priority, our spectroscopic DO sample is likely to be fairly complete within the volume surveyed by the SDSS. The DO stars cover the left bottom corner of the diagram and are mixed with the hot subdwarf, DB, and DA stars, so it is not possible to separate DO stars from other white dwarf/subdwarf stars using photometry measurements alone; we must also have spectra.

\section{Spectra and photometric parameters}

Table 1 summarizes the photometric and astrometric data for all our 15 DO white dwarf stars, 13 of which are contained 
Table 1. Photometry and proper motion of SDSS DO white dwarf stars.

\begin{tabular}{|c|c|c|c|c|c|c|c|c|c|c|c|}
\hline No. & Star(SDSS+) & MJD Plt-Fib & $u$ & $g$ & $r$ & $i$ & $z$ & $\begin{array}{r}\mathrm{PM}_{\mathrm{RA}} \\
{[\mathrm{mas} / \mathrm{yr}]}\end{array}$ & $\begin{array}{r}\mathrm{PM}_{\mathrm{Dec}} \\
{[\mathrm{mas} / \mathrm{yr}]}\end{array}$ & $\begin{array}{l}\text { Spec } \\
\text { type }\end{array}$ & Note \\
\hline 1 & J001651.41-011329.4 & 51795 389-041 & 16.08 & 16.58 & 17.04 & 17.37 & 17.81 & 5 & -18 & PG 1159 & $\mathrm{E}$ \\
\hline 2 & $\mathrm{~J} 114635.23+001233.5$ & $51959283-502$ & 14.36 & 14.88 & 15.42 & 15.83 & 16.19 & -1 & -7 & PG 1159 & $\mathrm{E}$ ah \\
\hline 3 & $\mathrm{~J} 134341.89+670154.5$ & $51989497-442$ & 16.33 & 16.87 & 17.45 & 17.81 & 18.15 & -12 & -1 & PG 1159 & A \\
\hline 4 & J075540.94+400918.0 & $51882435-109$ & 17.01 & 17.49 & 18.06 & 18.43 & 18.78 & 3 & 1 & PG 1159 & A \\
\hline 5 & $\mathrm{~J} 151026.47+610657.0$ & $52055611-070$ & 16.44 & 16.95 & 17.57 & 17.93 & 18.36 & -3 & -4 & DO & uhei \\
\hline 6 & $\mathrm{~J} 025403.76+005854.3$ & $52175708-448$ & 16.53 & 17.02 & 17.52 & 17.89 & 18.14 & 7 & -3 & DO & uhei * \\
\hline 7 & $\mathrm{~J} 140409.95+045740.0$ & $52045582-430$ & 16.92 & 17.46 & 17.97 & 18.33 & 18.65 & 6 & -14 & DO & \\
\hline 8 & $\mathrm{~J} 155356.81+483228.6$ & $52352812-187$ & 17.90 & 18.37 & 18.87 & 19.29 & 19.79 & 2 & -33 & DO & $*$ \\
\hline 9 & J091433.60+581238.1 & $51907484-151$ & 17.12 & 17.59 & 17.98 & 18.20 & 18.32 & 1 & 4 & DO & \\
\hline 10 & $\mathrm{~J} 131724.75+000237.3$ & $51985295-545$ & 15.29 & 15.77 & 16.32 & 16.70 & 17.07 & -15 & 13 & DO & bh \\
\hline 11 & J034101.39+005352.9 & $51811416-458$ & 17.49 & 17.84 & 18.25 & 18.57 & 18.80 & 1 & -6 & DO & \\
\hline 12 & J034227.63-072213.3 & $51909462-087$ & 17.29 & 17.64 & 18.13 & 18.49 & 18.81 & -1 & -8 & DO & $\mathrm{h}$ \\
\hline 13 & J003343.06+142251.5 & $51821417-104$ & 17.24 & 17.56 & 18.01 & 18.33 & 18.53 & 36 & -7 & DBAO & \\
\hline 14 & J015629.60+131744.8 & $51900427-320$ & 17.95 & 18.10 & 18.38 & 18.60 & 18.86 & 8 & -4 & DBO: & $\mathrm{c}$ \\
\hline 15 & J163200.34-001928.3 & $51671348-310$ & 18.01 & 18.41 & 18.83 & 19.16 & 19.49 & -3 & -3 & DAO & $\mathrm{h}$ \\
\hline
\end{tabular}

A subtype A of PG 1159 type stars (Werner 1992).

${ }^{\mathrm{E}}$ subtype E of PG 1159 type stars (Werner 1992).

${ }^{a}$ known as WD1144+004 or PG1144+005.

${ }^{\mathrm{b}}$ known as WD1314+003 or HE1314+0018.

${ }^{\mathrm{c}}$ object was classified as an uncertain DBO (DBO:) in Kleinman et al. (2003).

$\mathrm{h}$ included in Harris et al. (2003).

uhei spectrum shows ultra-high excitation ion features.

* spectrum does not exist in DR1.

in the DR1 database. Positions at the epoch of observation are taken from the SDSS Astrometric Pipeline (Pier et al. 2003). MJD Plt-Fib denotes the modified Julian date of observation, plate number and fiber number assigned for each object $^{3}$. The SDSS $u, g, r, i, z$ magnitudes are SDSS PSF magnitudes from the SDSS Photometric Pipeline version 5.3 (Lupton et al. 2003). We do not tabulate the magnitude uncertainties in Table 1 , but these are typically of order $0.01 \mathrm{mag}$. The next two columns contain the proper motion in right ascension and declination in milli arc seconds per year. The photometric calibration is based on the SDSS standard-star system (Smith et al. 2002), and proper motions are calculated using the USNO-B1.0 catalog (Monet et al. 2003; see also Kleinman et al. 2004 and Munn et al. 2003, for a more detailed description of the proper motion calculation). Figures $2-4$ show the SDSS spectra within the wavelength range from $3830 \AA$ to $6650 \AA$ for each object in Table 1. Each plot uses the same scale and were prepared in the same way: first, each spectrum was smoothed by 2-7 pixels (depending on the line feature visibility and/or noise) and then normalized by a highly smoothed (300 pixel) version of itself. To help identify the individual line positions, the Balmer

\footnotetext{
${ }^{3}$ An SDSS spectrum is uniquely described by its MJD, Plate, and Fiber. However the official way to refer to an SDSS object is by the name given in the second column of Table 1.
}

series of hydrogen $(\mathrm{H})$, helium I $\left(\mathrm{He}_{\mathrm{I}}\right)$ and helium II $\left(\mathrm{He}_{\mathrm{II}}\right)$ lines are plotted along with the spectra. We also include lines corresponding to certain features visible in different subtypes of DO spectra. Following SDSS convention, all SDSS spectra are converted into vacuum wavelength using the formula given by Morton (1991). The differences between air and vacuum line positions are small (less than $2 \AA$ within the wavelength range shown), therefore difficult to notice in the plots. Whenever we are referring to a certain wavelength position or presenting a table containing wavelengths, however, we use the laboratory wavelength and not the vacuum wavelength.

\subsection{PG 1159 stars}

Among our list of DO stars, there are four PG 1159 type stars - the hotter extension of DO white dwarf sequence and DO progenitors - including a previously known PG 1159 star, 51959 283-502, also known as WD1144+004 and PG 1144+005 (Werner \& Heber 1991). They each show typical $\mathrm{C}_{\mathrm{IV}}$ features in the 4650-4690 $\AA$ region and a lack of hydrogen or helium I lines (see Fig. 2). As is clearly visible in the figure, the top two stars, 51795 389041 and 51959 283-502, show emission of $\mathrm{N}_{\mathrm{V}} 4945 \AA$, $\mathrm{O}_{\mathrm{VI}} 5291 \AA, \mathrm{C}_{\mathrm{IV}} 5801 / 5812 \AA$, as well as emission line cores, 


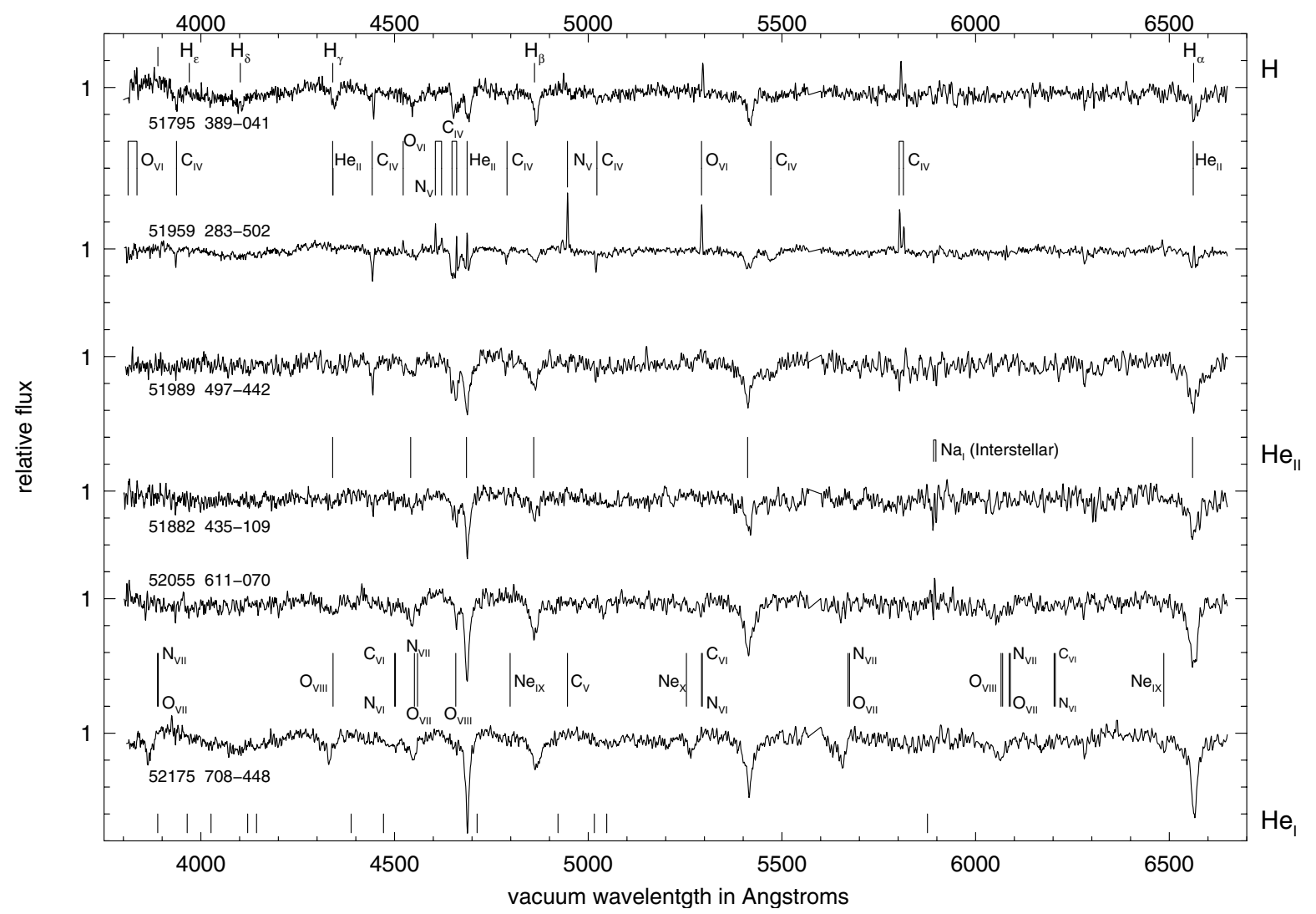

Fig. 2. PG 1159 and DO ultra-high excitation ion (uhei) feature spectra (the display order in the plot corresponds to the order in Table 1). From the top: (MJD Plt-Fib) 51795 389-041: PG 1159 type (subtype E: see Werner 1992 for subtype discussion), 51959 283-502: PG 1159 type (subtype E) also known as PG 1144+005 or WD1144+004, 51989 497-442: PG 1159 type (subtype A), 51882 435-109: PG 1159 type (subtype A), 52055 611-070: DO uhei star, and 52175 708-448: DO uhei star. In addition to $\mathrm{H}, \mathrm{He}_{\mathrm{I}}$ and $\mathrm{He}_{\mathrm{II}}$ line series, lines characteristic for PG 1159 E subtype stars are indicated near the top of the figure, along with the uhei features in the bottom part of the figure. The continua of all spectra have been normalized to unity and plotted with the same flux scale; tick marks are given at intervals of 0.1 .

particularly pronounced in the latter spectrum $\left(\mathrm{He}_{\text {II }} 4686 \AA\right.$ and $\mathrm{He}_{\text {II }} 6560 \AA$ ). The spectral features for $51959283-502$ star have already been identified and discussed by Werner \& Heber (1991). We marked the identified spectral features from their paper in Fig. 2 as the series of lines between the spectra of 51795 389-041 and 51959 283-502. The fainter of the two PG 1159 stars, 51795 389-041 (the top spectrum in Fig. 2) is very similar to $51959283-502$, showing a weak emission core at $\mathrm{He}_{\text {II }} 6560 \AA$ and perhaps at $\mathrm{He}_{\text {II }} 4686 \AA$ as well (but this needs to be verified by future observations). So, 51795 389-041 and 51959 283-502 can be classified as subgroup E of PG 1159 type stars (Werner 1992) ${ }^{4}$. Werner \& Heber (1991) estimated the temperature and gravity for $51959283-502$ to be: $T_{\text {eff }} \approx 150000 \mathrm{~K}$ and $\log g \approx 6.5$. The similarity of 51795 389-041's spectral features to those of 51959 283-502 suggests both stars are similar in temperature. We failed to find evidence for planetary nebula remnants around both 51795 389-041 and 51959 283-502, but the short effective integration time of SDSS images (30 s) results in the inefficient detection of very faint extended objects.

The next two spectra in Fig. 2, $51989497-442$ and 51882 435-109, show PG 1159-characteristic $\mathrm{C}_{\mathrm{IV}}$ absorption

\footnotetext{
${ }^{4}$ Subgroup type $\mathrm{E}$ (emission) exhibits emission cores in $\mathrm{He}_{\mathrm{II}} 4686 \AA, \mathrm{C}_{\mathrm{IV}} 4659 \AA$, and weak $\mathrm{C}_{\mathrm{IV}} 4647 \AA$.
}

lines near 4647/4659 $\AA$, but since no emission lines are visible, we classified them as subgroup A of PG $1159 \mathrm{stars}^{5}$. According to Werner (1992), objects like these are relatively cool: i.e. $T_{\text {eff }} \lesssim 100000 \mathrm{~K}$.

\subsection{DO stars showing ultra-high excitation ion features}

We also found ultra-high excitation ion (uhei) absorption line features of $\mathrm{C}_{\mathrm{VI}}, \mathrm{N}_{\mathrm{VII}}, \mathrm{O}_{\mathrm{VII}}, \mathrm{O}_{\mathrm{VIII}}, \mathrm{Ne}_{\mathrm{IX}}$ and maybe $\mathrm{Ne}_{\mathrm{X}}$ in the next two DO star spectra of Fig. 2: 52055 611-070 and $52175708-448^{6}$. These features have been previously found and described in six other stars by Werner et al. (1995) and Dreizler et al. (1995). According to their work, the temperatures required for such highly excited ion production is higher than $500000 \mathrm{~K}$. While it is possible for a massive post-AGB remnant star to reach that range of temperature (a $1.2 M_{\odot}$ postAGB star's effective temperature can reach up to $700000 \mathrm{~K}$ at maximum, Paczynski 1970) for a very short time, Werner's et al. (1995) model calculations show that their uhei features

\footnotetext{
${ }^{5}$ Subgroup type A (absorption) shows pure absorption in the entire spectrum.

${ }^{6}$ Due to its narrow spectral lines, $52175708-448$ could also be a subdwarf O star.
} 
Table 2. Ultra-high excitation ion lines identified in two DO uhei star spectra.

\begin{tabular}{|c|c|c|c|}
\hline Ion & $\begin{array}{c}\text { Transition } \\
\lambda[\AA]\end{array}$ & $\begin{array}{c}\text { SDSS } \\
52055611-070\end{array}$ & $\begin{array}{c}\text { SDSS } \\
52175708-448\end{array}$ \\
\hline $\mathrm{C}_{\mathrm{V}}$ & 4945 & & \\
\hline \multirow{3}{*}{$\mathrm{C}_{\mathrm{VI}}$} & 4499 & & maybe \\
\hline & 5291 & maybe & $\mathrm{X}$ \\
\hline & 6201 & & maybe \\
\hline \multirow[t]{3}{*}{$\mathrm{N}_{\mathrm{VI}}$} & 4501 & & maybe \\
\hline & 5293 & maybe & X \\
\hline & 6204 & & maybe \\
\hline \multirow[t]{4}{*}{$\mathrm{N}_{\mathrm{VII}}$} & 3887 & & X \\
\hline & 4550 & $X$ & $X$ \\
\hline & 5669 & $X$ & $X$ \\
\hline & 6085 & $X$ & $X$ \\
\hline \multirow[t]{4}{*}{$\mathrm{O}_{\mathrm{VII}}$} & 3889 & & $X$ \\
\hline & 4558 & $X$ & $X$ \\
\hline & 5673 & $X$ & $X$ \\
\hline & 6088 & $X$ & $X$ \\
\hline \multirow[t]{4}{*}{$\mathrm{O}_{\mathrm{VIII}}$} & 4340 & $X$ & $X$ \\
\hline & 4658 & $\mathrm{X}$ & maybe \\
\hline & 6064 & maybe & maybe \\
\hline & 6068 & maybe & maybe \\
\hline \multirow[t]{2}{*}{$\mathrm{Ne}_{\mathrm{IX}}$} & 4797 & & \\
\hline & 6484 & & $X$ \\
\hline $\mathrm{Ne}_{\mathrm{X}}$ & 5252 & & maybe \\
\hline
\end{tabular}

cannot be of photospheric origin. Models capable of producing the uhei lines failed to accurately reproduce the normal $\mathrm{He}_{\mathrm{II}}$ lines. Werner \& Dreizler propose a possible explanation for the uhei lines is that they are formed in a hot stellar wind, very close to the stellar surface (not more than 1 stellar radius away from the surface so that no emission lines are visible). In support of this explanation is the fact that the cores of the uhei lines are blue-shifted compared to their rest wavelengths. One can notice that our two uhei feature stars shown in Fig. 2 also have uhei lines that are blue-shifted. In addition, the lines apear to be asymmetric as were those presented in Werner et al. (1995) and Drieizler et al. (1995) papers, which is to be epected from wind-based origin. Unfortunately, because of the low resolution of the SDSS spectra, all highly excited ion lines are either blended with other nearby lines or just too noisy to be used for precise radial velocity estimations.

Table 2 contains the uhei feature line identification of 52055 611-070 and 52175 708-448, presented in a similar way to those presented by Werner et al. (1995) and Dreizler et al. (1995). These uhei lines have also been indicated in Fig. 2 as the series of lines in between two uhei star spectra. The feature near 4647/4659 $\AA$ seen in 52055 611-070 is near the characteristic $\mathrm{C}_{\mathrm{IV}}$ lines of $\mathrm{PG} 1159$ stars, but since we do not see both components of the normal $\mathrm{C}_{\mathrm{IV}}$ doublet, this line may instead be from the nearby $\mathrm{O}_{\mathrm{VIII}}$. However, if it is $\mathrm{O}_{\mathrm{VIII}}$, we cannot explain why it is not blue-shifted like the other uhei lines are, nor why its shape does not recall those of the other uhei lines. In the end, we cannot say with any certainty the origin of this line.

\subsection{DO stars}

Figure 3 shows the six normal DO star spectra from Table 1. The top three are ordered by their dereddened $u-i$ colors as indicated by the $(u-i)_{0}$ values below each spectrum. The next three spectra, where $\mathrm{He}_{\mathrm{I}}$ lines become visible, are ordered by the relative strength of the $\mathrm{He}_{\text {II }}$ lines $4686 \AA, 5412 \AA$ and $4542 \AA$ compared to the $\mathrm{He}_{\mathrm{I}}$ lines $4472 \AA, 5876 \AA$, and those between 4800-5100 ̊. Broadband color differences can be reasonable temperature indicators for hot white dwarf stars. The apparent line strengths of the $\mathrm{He}_{\mathrm{II}}$ lines of the objects in Fig. 3, however, suggest a different temperature order than that which their boradband colors would suggest. We have therefore ordered the spectra by their apparent line strengths as an indication of temperature. Lacking a proper grid of detailed atmospheric models, we are not sure of the correct temperature ordering of these stars, so provide the $(u-i)_{0}$ colors alongside each spectrum as well. The brightest star in this group, 51985 295-545, is already known as WD1314+003 and HE1314+0018 (Christlieb et al. 2001) and was listed (as well as with 51909 462-087) in Harris et al. (2003). Only these two stars, along with $51811416-458$, have $\mathrm{He}_{\mathrm{I}}$ lines along with $\mathrm{He}_{\mathrm{II}}$ lines. In the case of $51909462-087$, the $\mathrm{He}_{\mathrm{I}}$ line around $4471 \AA$ is probably stronger than the $4686 \AA \mathrm{He}_{\text {II }}$ line (however the difference in depth of these two lines is not statisticaly significant), but this indicates that the star is probably the coolest in this set of DO stars with a temperature $\$ 50000 \mathrm{~K}$.

\section{4. $D B O, D B A O$ and $D A O$ stars}

We have also found three new hybrid DBO, DBAO and DAO stars, where the naming convention is the one suggested by Wesemael et al. (1993) and which has been explained in Sect. 1. Such stars look like their normal DA and DB counterparts, except for the presence of the $\mathrm{He}_{\text {II }}$ (mostly $4686 \AA$ ) lines, which earns them their $\mathrm{O}$ designations. These types of stars are interesting from an evolutionary standpoint since all DO stars after their short, hot lives, must become cooler DB or DA stars. There must therefore be an intermediate state during which the $\mathrm{He}_{\mathrm{II}}$ lines are weakening and $\mathrm{He}_{\mathrm{I}}$ and/or $\mathrm{H}$ lines are strengthening. Also, the analysis of DBO stars might place a new limit on the hot end of the DB gap (see Liebert 1985; Liebert et al. 1986). In Fig. 4, we show the spectra of our 3 hybrid objects: the DBAO 51821 417-104 and DBO: 51900 427-320 along with the DAO 51671 348-310 (also in Harris et al. 2003). Only a weak $4686 \AA \mathrm{He}_{\text {II }}$ line remains visible in all these spectra, while the other $\mathrm{He}_{\mathrm{II}}$ lines are blended or dominated by hydrogen lines. Since we are not certain if $\mathrm{He}_{\mathrm{II}} 4686 \AA$ line in 51900 427-320 star spectra is significant or not, we added a ":" sign to its DBO name as a measure of uncertainty. The DAO, 51671 348-310, has strange absorption line profiles. We suspect they may be indicating the presence of a surface magnetic field, but cannot be certain without more observations. The apparent splitting seen is consistent with a mean surface field of a 


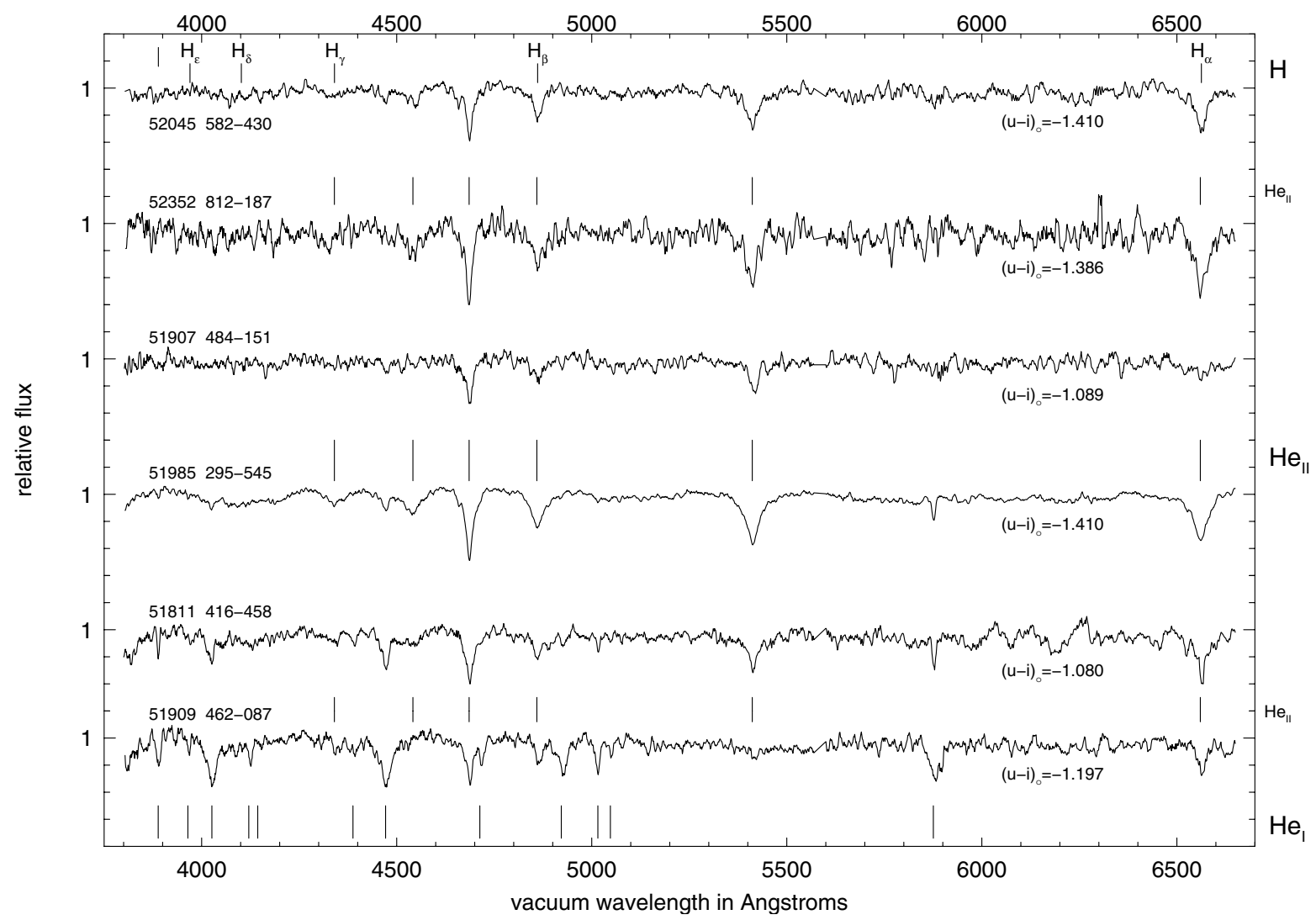

Fig. 3. "Normal" DO stars observed in the SDSS survey. The brightest one, 51985 295-545, is already known as WD1314+003 and HE 1314+0018. This and $51909462-087$ have already been presented in Harris et al. (2003). Dereddened $u-i$ color differences are shown below each spectrum. The continua of all spectra have been normalized to unity and plotted with the same flux scale; tick marks are given at intervals of 0.1 .

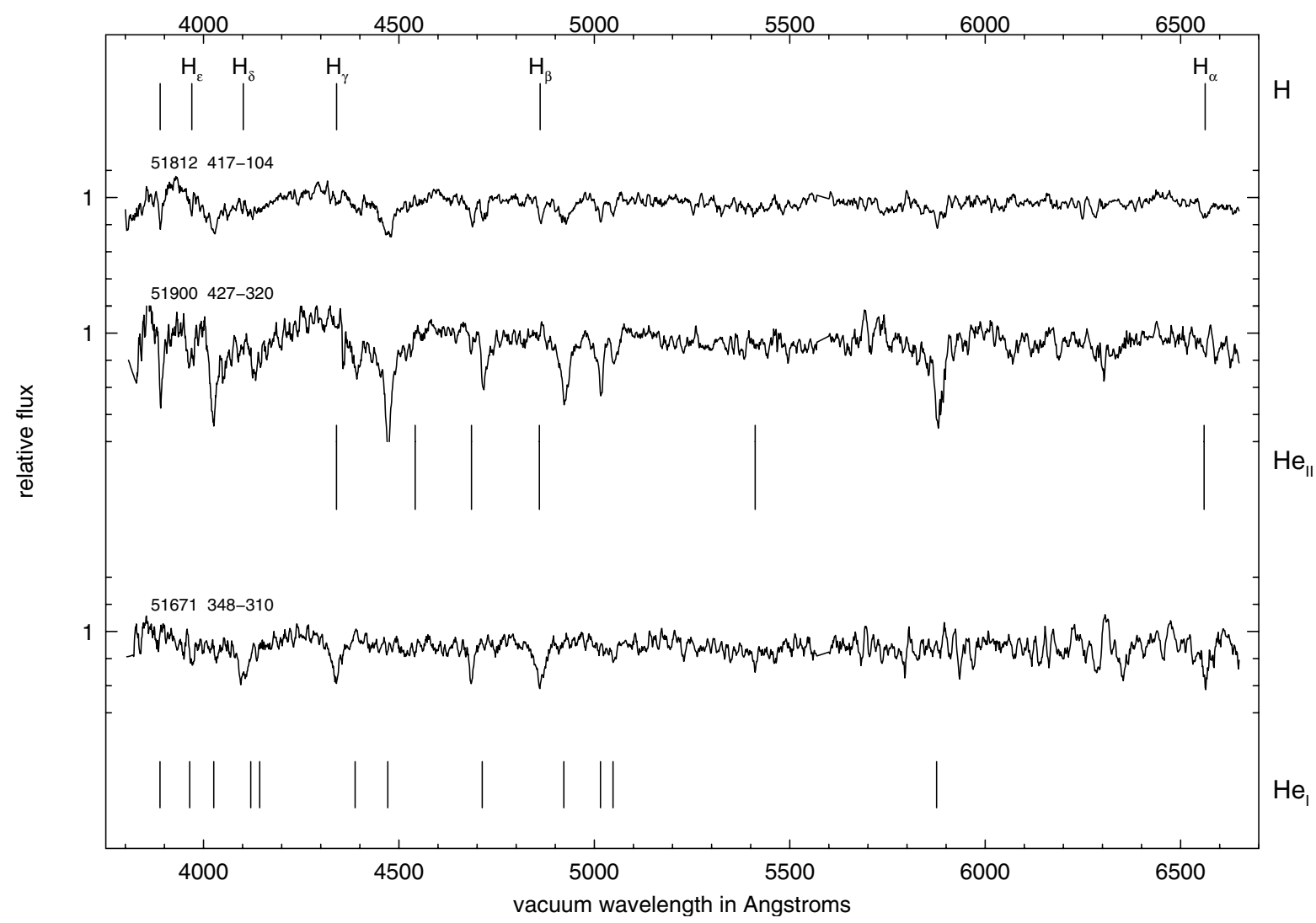

Fig. 4. New SDSS DBO and DBAO (first top two spectra) and DAO (bottom spectrum) stars. The continua of all spectra have been normalized to unity and plotted with the same flux scale; tick marks are given at intervals of 0.1 . 
little less than $1 \mathrm{MG}$ (SDSS spectrographs with their resolution power of $\sim 1800$ is effective for recognizing the Zeeman effect for $B>1-3 \mathrm{MG}$, Schmidt et al. 2003). But interestingly, there is no evidence of splitting at $\mathrm{He}_{\mathrm{II}} 4686 \AA$, so that might argue against the magnetic field interpretation.

\section{Conclusions}

In the first SDSS data release, there were $\approx 2500$ new white dwarf stars discovered, including 13 DO stars of various subtypes. Our sample adds two additional DO stars observed early in the SDSS, but not yet released to the public, for a total of 15 DO stars - 13 of which are new. This portends a very promising future since not only will the survey double the number of currently known DO stars by its end, but we will also gain knowledge about their distribution in the Galaxy. Thanks to the relatively high priority of the SDSS HOT_STD targeting category, the resultant set of new DO stars is likely to be quantitatively complete to an ascertainable level. Such a sample will provide new insights into the formation and evolution of the DO stars and their descendants and predecessors.

There are still various questions which have to be answered concerning hot white dwarf evolution. Which stars are progenitors of PG 1159 type stars and which are descendants? It seems that PG 1159 stars must turn into DO stars and that DO stars should then become DB and DA stars. A more complete sample of DOs, including some of the more rare, shorter-lived, subtypes should help us understand this evolutionary process. The uhei DOs are still a mystery themselves. To the current sample of 6 known uhei DO stars, this work adds two more. At this rate, the SDSS should add a total of 10 or more by survey end. The future is indeed bright for the study of the hottest white dwarf stars.

Acknowledgements. Funding for the Sloan Digital Sky Survey (SDSS) has been provided by the Alfred P. Sloan Foundation, the Participating Institutions, the National Aeronautics and Space Administration, the National Science Foundation, the US Department of Energy, the Japanese Monbukagakusho, and the Max Planck Society. Participating Institutions: the SDSS is a joint project of The University of Chicago, Fermilab, the Institute for Advanced Study, the Japan Participation Group, The Johns Hopkins University, Los Alamos National Laboratory, the Max-Planck-Institute for
Astronomy (MPIA), the Max-Planck-Institute for Astrophysics (MPA), New Mexico State University, University of Pittsburgh, Princeton University, the United States Naval Observatory, and the University of Washington.

We thank Stefan Dreizler for pointing out the existence of the high excitation lines in DOs and useful conversation on these objects. J.K., A.N. and S.J.K. also would like to thank J. Peoples and B. Gillespie for their support of this research program.

\section{References}

Abazajian, K., Adelman-McCarthy, J. K., Agüeros, M. A., et al. 2003, AJ, 126, 2081

Barstow, M. A., \& Holberg, J. B. 1990, MNRAS, 245, 370

Bergeron, P., Wesemael, F., \& Beauchamp, A. 1995, PASP, 107, 1047

Christlieb, N., Wisotzki, L., Reimers, D., et al. 2001, A\&A, 366, 898

Dreizler, S., Heber, U., Napiwotzki, R., \& Hagen, H. J. 1995, A\&A, 303, L53

Dreizler, S., \& Werner, K. 1996, A\&A, 314, 217

Harris, C. H., Liebert, J., Kleinman, S. J., et al. 2003, AJ, 126, 1023

Kleinman, S., et al. 2004, in preparation

Liebert, J. 1985, The origin and evolution of helium-rich white dwarfs, in Hydrogen Deficient Stars and Related Objects, ed. K. Hunger, \& K. Rao (Dordrecht: D. Tedidel Publishing Co.), Proc. IAU Coll., 87, 367

Liebert, J., Wesemael, F., Hansen, C. J., et al. 1986, ApJ, 309, 241

Lupton, R., et al. 2003, in preparation

McCook, G. P., \& Sionthe, E. M. 1999, ApJS, 121, 1

Monet, D. G., et al. 2003, AJ, in press

Morton, D. C. 1991, ApJS, 77, 119

Munn, J., et al. 2003, in preparation

O’Brien, M. S., \& Kawaler, S. D. 2000, ApJ, 539, 372

Paczynski, B. 1970, Acta Astr., 20, 47

Pier, J. R., Munn, J. A., Hindstey, R. B., et al. 2003, AJ, 125, 1559

Schlegel, D. J., Finkbeiner, D. P., \& Davis, M. 1998, ApJ, 500, 525

Schmidt, D. G., et al. 2003, ApJ, 595, 1101

Sion, E. M., Greenstein, J. L., Landstreet, J. D., et al. 1983, AJ, 269, 253

Smith, J. A., Tucker, D. L., Kent, S., et al. 2002, AJ, 123, 2121

Werner, K. 1992, in The Atmospheres of Early-Type Stars, ed. U. Heber, \& C. S. Jeffery (Berlin, New York: Springer-Verlag), 273

Werner, K., Dreizler, S., Heber, U., et al. 1995, A\&A, 293, L75

Werner, K., \& Heber, U. 1991, A\&A, 247, 476

Wesemael, F., Greenstein, J. L., Liebert, J., et al. 1993, PASP, 105, 761

York, D. G., Adelman, J., Anderson, J. E. Jr., et al. 2000, AJ, 120, 1579 